\title{
Ultrafast critical dynamics of a ferroelectric phase transition in $\mathrm{Pb}_{1-x} \mathrm{Ge}_{x} \mathrm{Te}$
}

\author{
Rong Lu, ${ }^{1,2}$ Muneaki Hase, ${ }^{1,3,4, *}$ Masahiro Kitajima, ${ }^{1,4}$ Shinichi Nakashima, ${ }^{5}$ and Shunji Sugai ${ }^{6}$ \\ ${ }^{1}$ Advanced Nano-Characterization Center, National Institute for Materials Science, 1-2-1 Sengen, Tsukuba 305-0047, Japan \\ ${ }^{2}$ Department of Chemistry, Renmin University of China, 59 Zhongguancun Avenue, Beijing, 100872, China \\ ${ }^{3}$ PRESTO, Japan Science and Technology Agency, 4-1-8 Honcho, Kawaguchi, Saitama 332-0012, Japan \\ ${ }^{4}$ Institute of Applied Physics, University of Tsukuba, Tennodai, Tsukuba, 305-8573, Japan \\ ${ }^{5}$ Power Electronics Research Center, National Institute of Advanced Industrial Science and Technology, 1-1-1 Umezono, Tsukuba \\ 305-8568, Japan \\ ${ }^{6}$ Department of Physics, Faculty of Science, Nagoya University, Furo-cho, Chikusa-ku, Nagoya 464-8602, Japan
}

(Received 6 October 2006; revised manuscript received 4 December 2006; published 23 January 2007; publisher error corrected 24 January 2007)

\begin{abstract}
Transient responses of the electronic excitation and coherent soft phonon are investigated both above and below the ferroelectric phase transition temperature $T_{c}$ in $\mathrm{Pb}_{1-x} \mathrm{Ge}_{x} \mathrm{Te}$ by using an optical pump-probe technique. The coherent soft mode shows large redshift and heavily overdamped decay as the temperature approaching $T_{c}$ from the low temperature side, and the soft mode disappears above $T_{c}$. The transient electronic response exhibits an abrupt change across $T_{c}$. The critical behaviors of both the phononic and electronic dynamics are interpreted by the ferroelectric phase transition.
\end{abstract}

DOI: 10.1103/PhysRevB.75.012107

Until now optical rewritable memory media commonly used in commercial disks is $\mathrm{Ge}_{2} \mathrm{Sb}_{2} \mathrm{Te}_{3}$ (GST) system, ${ }^{1}$ in which the laser-induced phase change between crystalline and amorphous phases predominates the recording process. The rapid phase transition time in GST was found to be several nanoseconds range. On the other hand, utilizing the reversal of ferroelectric domains by ultrashort optical pulses, optical data storage or optical switching with time scale of less than 10 picoseconds has been proposed. ${ }^{2}$ In this optical switch, one may switch the orientation of ferroelectric domains within a few phonon periods in time using femtosecond laser excitation, where the phonon displacement reaches a critical displacement of an order of $1 \%$ of the interatomic distance. ${ }^{3,4}$

Ferroelectric structural phase transition has been extensively investigated by monitoring the soft mode, i.e., the lowest frequency transverse optical (TO) phonon, whose zone center frequency $\omega_{\mathrm{TO}}$ is drastically reduced toward zero near the critical point $T_{c}{ }^{5,6}$ By using frequency domain techniques, such as Raman and neutron scattering, temperature dependence of $\omega_{\mathrm{TO}}$ has been examined, ${ }^{6}$ although the observation of the soft mode near $T_{c}$ is very difficult because of the extremely low frequency and broad linewidth of the soft mode.

Motivated by the observation of real-time dynamics of ferroelectric phase transitions, a few researches have examined femtosecond pump-probe measurements of the coherent soft modes in order-disorder type perovskites, such as $\mathrm{KNbO}_{3}$ (Ref. 7) and $\mathrm{SrTiO}_{3},{ }^{8}$ and in displacive type ferroelectric semiconductor GeTe. ${ }^{9}$ The generation mechanism of the coherent soft mode in perovskites was considered to be nonresonant impulsive stimulated Raman scattering (NR-ISRS), ${ }^{7}$ while in optically absorbing media, like GeTe, the coherent phonon can be generated by resonant impulsive stimulated Raman scattering (R-ISRS) ${ }^{10}$ which is recognized to be the general case of the displacive excitation of coherent phonons (DECP) mechanism. ${ }^{11}$ Note that those pump-probe measurements were not done at the higher temperature than $T_{c}$ because the soft mode becomes Raman in-
PACS number(s): 78.47.+p, 63.20.-e, 77.80.Bh

active due to the symmetry changes and thus it will disappear at $T>T_{c}$.

Femtosecond pump-probe reflectivity technique has also been applied to observing coherent phonon dynamics in GST film by Först et al. ${ }^{12}$ They observed drastic changes of the coherent optical phonon properties across the phase transition temperatures, and concluded that there was a metastable phase between the crystalline and amorphous phases. Until now, however, the time-domain study of the soft mode and electronic transient responses associated with the ferroelectric phase transition at the temperatures both above and below $T_{c}$ is still missing.

In this paper, we investigate both the coherent soft phonon and transient electronic response in $\mathrm{Pb}_{1-x} \mathrm{Ge}_{x} \mathrm{Te}$ (PGT) utilizing a femtosecond optical pump-probe technique in a wide range of the lattice temperature. The ferroelectric material PGT is a narrow band-gap semiconductor $\left(E_{g} \sim 0.3 \mathrm{eV}\right)$ with a high carrier mobility and is useful for infrared laser and detectors. ${ }^{13}$ It exhibits structural phase change from rhombohedrally distorted structure below $T_{c}$ to cubic rocksalt structure above $T_{c}$, and $T_{c}$ depends on the composition $x$, promising that one can control $T_{c}$ by changing $x .{ }^{14-16}$ The spectroscopic measurements revealed that the band-gap energy in PGT is very sensitive to the ferroelectric phase transition, ${ }^{16}$ suggesting that ultrafast dynamics of photogenerated carriers exhibit significant changes across $T_{c}$.

The time-resolved reflectivity measurement was performed on a single crystal of $\mathrm{Pb}_{1-x} \mathrm{Ge}_{x} \mathrm{Te}(x=0.07)$ obtained by Bridgman method. At this composition $x, T_{c}$ is expected to be $160-170 \mathrm{~K} .{ }^{15}$ In PGT the TO phonons $\left(A_{1}\right.$ and $E$ symmetries) are soft modes, whose displacement is along the cubic (111) direction $\left(A_{1}\right.$ mode) and is perpendicular to the cubic (111) direction ( $E$ mode), and those modes are responsible for the phase transition. ${ }^{14}$ The light source used was a mode-locked Ti:sapphire laser amplified with a center wavelength of $800 \mathrm{~nm}(1.55 \mathrm{eV})$ and a pulse duration of $\sim 150 \mathrm{fs}$. The pump and probe beams were focused on the sample to a diameter of $\sim 70 \mu \mathrm{m}$ and the pump fluence $\left(F_{p}\right)$ was reduced 


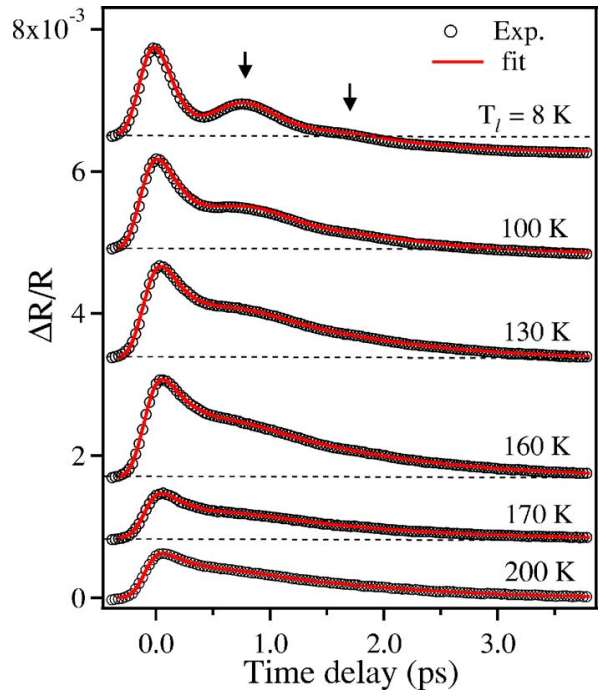

FIG. 1. (Color online) Transient reflectivity changes at the different $T_{l}$ as a function of the delay time. Open circles represent experimental data, and solid curves are the fitting using Eq. (1).

to below $0.1 \mathrm{~mJ} / \mathrm{cm}^{2}$ to prevent heating the sample. The probe fluence was fixed at $0.02 \mathrm{~mJ} / \mathrm{cm}^{2}$. The transient isotropic reflectivity change $(\Delta R / R)$ was recorded to observe the coherent $A_{1}$ mode as a function of the time delay at the lattice temperature $\left(T_{l}\right)$ from 8 to $200 \mathrm{~K}$.

Figure 1 shows the $\Delta R / R$ signal observed at various $T_{l}$ from 8 to $200 \mathrm{~K}$ at the constant fluence of $F_{p}=0.1 \mathrm{~mJ} / \mathrm{cm}^{2}$. The fast transient signal arising from the negative time delay represents linear electronic response with a few picoseconds relaxation time,${ }^{17}$ whereas the adjacent dip and the following coherent oscillation (indicated by arrows in Fig. 1) is the contribution from the coherent lattice vibration. The frequency of the coherent lattice vibration is $\approx 1.0 \mathrm{THz}$ $\left(=33 \mathrm{~cm}^{-1}\right)$ at $8 \mathrm{~K}$. The Raman measurements showed that the peak frequency of the $A_{1}$ mode in $\mathrm{Pb}_{1-x} \mathrm{Ge}_{x} \mathrm{Te}$ with different composition of $x=0.05$ was $\approx 23 \mathrm{~cm}^{-1} .{ }^{14}$ The larger Ge composition of our sample $(x=0.07)$ would result in higher frequency of the $A_{1}$ mode, ${ }^{15}$ and in addition, we are using conventional isotropic reflectivity technique which dominates totally symmetric $A_{1}$ mode rather than the $E$ mode. ${ }^{18}$ Therefore, this coherent oscillation corresponds to the soft $A_{1}$ mode. It should be emphasized that the fast electronic transient drastically changes: the transient electronic amplitude suddenly decreases around $170 \mathrm{~K}$ as shown in Fig. 1. These features imply that the phase transition occurs between 160 and $170 \mathrm{~K}$, and this value is quite close to the $T_{c}$ value reported by Raman scattering at the same Ge composition. ${ }^{15}$

In order to subtract the transient electronic response from the time-domain data and to obtain only coherent soft phonon, we utilize the following function to fit the data:

$$
\frac{\Delta R(t)}{R_{0}}=H(t)\left[A e^{-t / \tau_{A}}+B e^{-t / \tau_{B}}+C e^{-\gamma t} \cos (\omega t+\phi)\right],
$$

where $H(\mathrm{t})$ is the Heaviside function convoluted with Gaussian to account for the finite time resolution. $A$ and $B$ are the
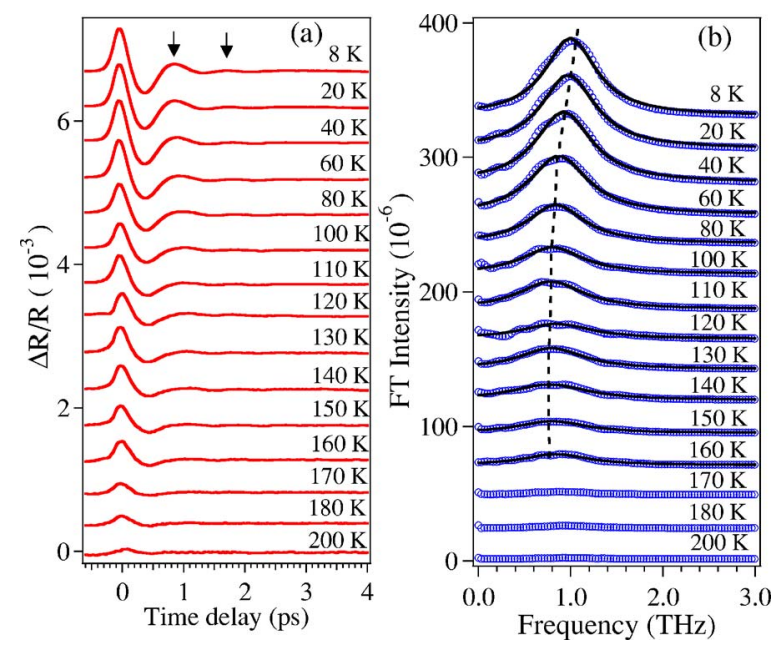

FIG. 2. (Color online) (a) Oscillatory part of the transient reflectivity at different $T_{l}$. The arrows indicate coherent soft mode. (b) The FT spectra obtained from the time-domain data in (a). Open circles represent experimental data, and solid curves are the fitting using Lorentz function. The dotted curve represents the peak frequency.

amplitudes, and $\tau_{A}$ and $\tau_{B}$ are the relaxation time of the fast and slow electronic responses, respectively. ${ }^{19} C, \gamma, \omega$, and $\phi$ are, respectively, the amplitude, damping rate, frequency, and initial phase of the underdamped soft $A_{1}$ phonon. As shown in Fig. 1, Eq. (1) fits the data very well at all $T_{l}$.

Figure 2(a) shows the oscillatory part of the changes of the reflectivity, which is obtained by extracting the exponential decay term from the above fitting at various $T_{l}$ from 8 to $200 \mathrm{~K}$ at the constant $F_{p}=0.1 \mathrm{~mJ} / \mathrm{cm}^{2}$. With increasing $T_{l}$, the coherent $A_{1}$ oscillation becomes weaker and almost vanishes at $T_{l} \approx 170 \mathrm{~K}$. This is explained by the fact that the $A_{1}$ mode is the soft mode, whose frequency reduces toward zero and damping becomes larger; we can detect only monocycle oscillation (the second cycle is rather weak) below $T_{c}$ $(=160-170 \mathrm{~K})$. Figure 2(b) represents the Fourier transformed (FT) spectra obtained from the time-domain data in (a). In Fig. 2(b), at $T_{l}=170 \mathrm{~K}$, the $A_{1}$ mode almost disappears, supporting the idea that $T_{c}$ is $160-170 \mathrm{~K}$. The FT spectra show the decrease in the peak frequency $\omega$ and the broadening of the linewidth of the coherent $A_{1}$ mode (this corresponds to the damping rate $\gamma$ ) as $T_{l}$ increases as shown in Fig. 3. The frequency $\omega$ gradually decreases with increasing $T_{l}$, while the damping $\gamma$ increases until $T_{l} \sim 140 \mathrm{~K}$. The broadening of the linewidth of the coherent phonon spectra with $T_{l}$ is explained by the enhanced anharmonic phononphonon coupling between the soft optical phonon and acoustic phonons, ${ }^{20}$ as observed in $\mathrm{Bi}^{21}$ The $T_{l}$ dependence of $\omega$ just below $T_{c}$ significantly deviates from the Landau's mean field theory; 22

$$
\omega=\alpha\left(T_{c}-T\right)^{\beta}
$$

which describes the second-order phase transition, where, amplitude $\alpha$ and exponent $\beta$ are the fitting parameters. As a result of the best fitting using the data at $T_{l} \leqslant 140 \mathrm{~K}, \alpha$ $=0.47$, and $\beta=0.14 \pm 0.02$ are obtained for the fixed value of 


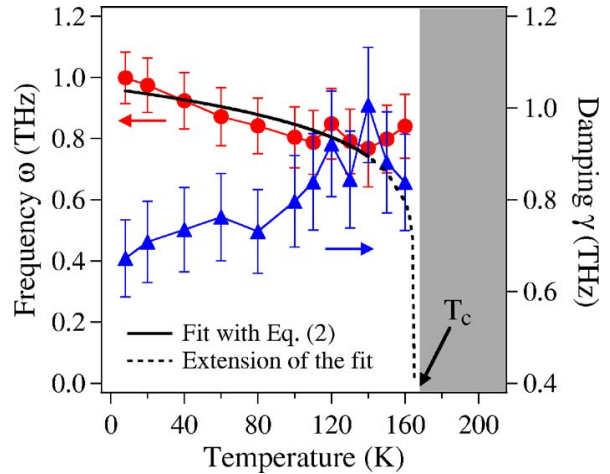

FIG. 3. (Color online) Temperature dependence of the frequency $\omega$ and the damping rate $\gamma$. The closed triangles and circles represent the experimental data. The solid curve is the fit of $\omega$ with Eq. (2) and the dotted curve represents extension of the fitting using Eq. (2) with the obtained parameters. The gray region at $T_{l} \geqslant 170 \mathrm{~K}$ represents the region in which no soft mode is available.

$T_{c}=165 \mathrm{~K}$. The deviation of the experimental $\omega$ from the Landau theory near $T_{c}$ suggests that the ferroelectric phase transition in PGT may be a mixture of the first-order and the second-order characters in the critical region due to the very large fluctuation amplitudes of the atoms. ${ }^{23}$

In Fig. 4, the amplitudes of the electronic transient and that of the coherent $A_{1}$ mode are plotted as a function of $T_{l}$ in order to discuss more details of the drastic change of the electronic transient as observed in Fig. $1 .{ }^{24}$ At $T_{l} \approx 165 \mathrm{~K}$ the electronic amplitude $\left(\Delta R / R_{e}\right)$ decreases from $1.2 \times 10^{-3}$ to $0.6 \times 10^{-3}$, which corresponds to $\sim 50 \%$ huge relative change. Since this huge change in $\Delta R / R_{e}$ occurs at the same temperature of the expected $T_{c}$ value, we interpret the drastic change in the fast electronic transient in terms of the result of ferroelectric phase transition. On the other hand, the $A_{1}$ mode amplitude $\left(I_{A 1}\right)$ gradually decreases with $T_{l}$, and becomes almost zero at $T_{l}=170 \mathrm{~K}$, being consistent with the ferroelectric character that the intensity of the soft mode becomes weak due to strong damping below $T_{c}$ and it vanishes due to the symmetry change at $T_{l} \geqslant T_{c}{ }^{6}$

The structural phase transition from ferroelectric phase $\left(T_{l}<T_{c}\right)$ to paraelectric phase $\left(T_{l}>T_{c}\right)$ accompanies displacement of the ions in the unit cell and modifies the ionic polarization: the spontaneous polarization below $T_{c}$ in the ferroelectric phase is large, while that above $T_{c}$ in the paraelectric phase vanishes. ${ }^{5}$ This ferroelectric phase transition then induces the changes in the electronic band structures, ${ }^{16}$ including the position of the acceptor levels. ${ }^{25}$ Based on the above considerations, possible origins for the drastic change in $\Delta R / R_{e}$ are considered as follows. First, the change in the electronic band structures would result in the change in the band gap via deformation potential (or more

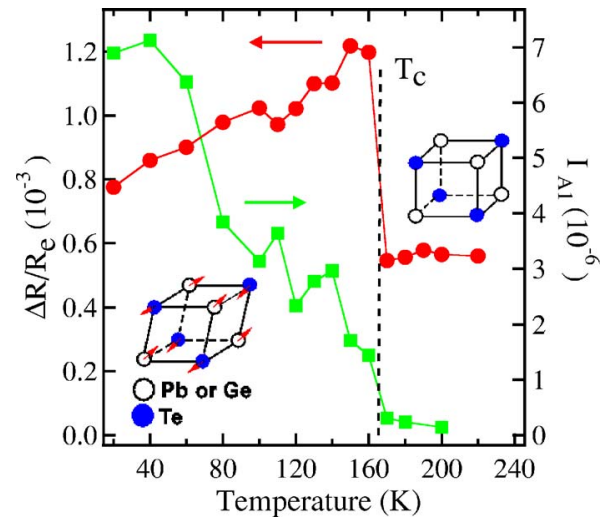

FIG. 4. (Color online) Temperature dependence of the amplitude of the electronic transient (closed circles) and that of the FT intensity of the coherent $A_{1}$ mode (closed squares) with solid guided lines. Below $T_{c}$, the arrows represent the displacement of the soft $A_{1}$ mode along (111) direction.

possibly piezoelectric effect due to spontaneous polarization) and also the change in the carrier mobility due to the electron-phonon interaction. ${ }^{26}$ These effects would change the interband optical absorption coefficient, and thus may change the electronic response $\Delta R / R_{e}$. Second, the change in the position of the acceptor levels affects the free carrier concentration, ${ }^{25}$ and this effect will result in the modification of the free carrier absorption, which contributes to the electronic response $\Delta R / R_{e}$. By using the huge change in the electronic transient $\Delta R / R_{e}$ across $T_{c}$, optical switching or optical memory using PGT can be proposed. In this case, photoinduced phase transition between ferroelectric and paraelectric phases is required. Such the photoinduced phase transition will be realized if one could precisely control both the amplitude ${ }^{27,28}$ and the frequency ${ }^{9}$ of the coherent soft phonon.

In summary, we have investigated ultrafast dynamics of both the coherent soft mode and the transient electronic polarization in ferroelectric semiconductor below and above $T_{c}$ by using the pump-probe technique. Low frequency coherent soft mode in PGT was observed only below $T_{c}$, showing the redshift of the frequency and heavily overdamped behavior as the temperature approaches $T_{c}$. The drastic decrease in the transient electronic response was revealed across $T_{c}$, suggesting the change in the electronic band structure, including the position of the acceptor levels.

The authors acknowledge J. Demsar and O. V. Misochko for helpful comments. This work was supported in part by a Grant-in-Aid for the Scientific Research from MEXT of Japan under Grant No. KAKENHI-16032218, and by Iketani Science Technology Foundation of Japan. 
*Electronic address: mhase@bk.tsukuba.ac.jp

${ }^{1}$ A. V. Kolobov, P. Fons, A. I. Frenkel, A. L. Ankudinov, J. Tominaga, and T. Uruga, Nat. Mater. 3, 703 (2004).

${ }^{2}$ S. Fahy and R. Merlin, Phys. Rev. Lett. 73, 1122 (1994).

${ }^{3}$ M. F. DeCamp, D. A. Reis, P. H. Bucksbaum, and R. Merlin, Phys. Rev. B 64, 092301 (2001).

${ }^{4}$ M. Hase, M. Kitajima, S. I. Nakashima, and K. Mizoguchi, Phys. Rev. Lett. 88, 067401 (2002).

${ }^{5} \mathrm{R}$. Brinc and B Zeks, Soft Modes in Ferroelectrics and Antiferroelectrics (North-Holland, Amsterdam, 1974).

${ }^{6}$ J. F. Scott, Rev. Mod. Phys. 46, 83 (1974).

${ }^{7}$ T. P. Dougherty, G. P. Wiederrecht, K. A. Nelson, M. H. Garrett, H. P. Jenssen, and C. Warde, Science 258, 770 (1992); , Phys. Rev. B 50, 8996 (1994).

${ }^{8}$ T. Kohmoto, K. Tada, T. Moriyasu, and Y. Fukuda, Phys. Rev. B 74, 064303 (2006).

${ }^{9}$ M. Hase, M. Kitajima, S. Nakashima, and K. Mizoguchi, Appl. Phys. Lett. 83, 4921 (2003).

${ }^{10}$ T. E. Stevens, J. Kuhl, and R. Merlin, Phys. Rev. B 65, 144304 (2002).

${ }^{11}$ H. J. Zeiger, J. Vidal, T. K. Cheng, E. P. Ippen, G. Dresselhaus, and M. S. Dresselhaus, Phys. Rev. B 45, 768 (1992).

${ }^{12}$ M. Först, T. Dekorsy, C. Trappe, M. Laurenzis, H. Kurz, and B. Béchevet, Appl. Phys. Lett. 77, 1964 (2000).

${ }^{13}$ T. Suski, M. Baj, and K. Murase, J. Phys. C 15, L377 (1982).

${ }^{14}$ K. Murase and S. Sugai, Solid State Commun. 32, 89 (1979).

${ }^{15}$ T. H. Hager, G. Bauer, and J. Brandmüller, J. Phys. C 19, 2153 (1986).
${ }^{16}$ S. Teraoka and K. Murase, J. Phys. Soc. Jpn. 51, 1857 (1982).

${ }^{17}$ The possible origins of the transient electronic response are interband transition above the band gap, and free carrier absorption in the valence bands $\left[\mathrm{Pb}_{1-x} \mathrm{Ge}_{x} \mathrm{Te}(x=0.07)\right.$ is considered to be p-type (Ref. 16)].

${ }^{18}$ T. Dekorsy, H. Auer, C. Waschke, H. J. Bakker, H. G. Roskos, H. Kurz, V. Wagner, and P. Grosse, Phys. Rev. Lett. 74, 738 (1995).

${ }^{19}$ The double exponential decay function in Eq. (1) is merely a matter of preference to subtract the electronic background and is not associated with some underlying relaxation mechanisms.

${ }^{20}$ J. Menendez and M. Cardona, Phys. Rev. B 29, 2051 (1984).

${ }^{21}$ M. Hase, K. Mizoguchi, H. Harima, S. I. Nakashima, and K. Sakai, Phys. Rev. B 58, 5448 (1998).

${ }^{22}$ Landau, Phys. Z. Sowjetunion 11, 26 (1937).

${ }^{23}$ K. A. Müller and W. Beringer, Phys. Rev. Lett. 26, 13 (1971).

${ }^{24} \mathrm{We}$ plot the amplitude of the electronic response by reading the maximum amplitude of $\Delta R / R_{e}(t)=H(t)\left(A e^{-t / \tau_{A}}+B e^{-t / \tau_{B}}\right)$ plotted by using the fitting parameters.

${ }^{25}$ L. Benguigui, R. Weil, A. Chack, E. Muranevich, and R. Nkum, Solid State Commun. 99, 619 (1996).

${ }^{26}$ P. Y. Yu and M. Cardona, Fundamentals of Semiconductors (Springer-Verlag, Berlin, Heidelberg, 1999), Chap. 3.

${ }^{27}$ M. Hase, K. Mizoguchi, H. Harima, S. Nakashima, M. Tani, K. Sakai, and M. Hangyo, Appl. Phys. Lett. 69, 2474 (1996).

${ }^{28}$ S. Iwai, Y. Ishige, S. Tanaka, Y. Okimoto, Y. Tokura, and H. Okamoto, Phys. Rev. Lett. 96, 057403 (2006). 